\title{
CONTEMPORARY SURGICAL TREATMENT OF THE OSTEOPOROTIC COMPRESSION FRACTURES
}

\author{
Svetoslav Kalevski ${ }^{1}$, Dimitar Haritonov ${ }^{1}$, Nikolay Peev ${ }^{1}$, Evgeniya Kalevska² \\ ${ }^{1}$ Department of Neurosurgery, Medical University of Varna, MHAT St. Anna, Varna \\ ${ }^{2}$ Department of Neurology, Medical University of Varna, UMHAT St. Marina, Varna
}

\begin{abstract}
AIM: The vertebral compression fractures (VCF) are among the most common complications of osteoporosis that could be a cause for permanent and debilitating pain, restricted mobility and hence - significant worsening of the quality of the life. The surgical treatment of VCF is indicated after a failure of the conservative treatment or after established spinal instability or neurological deficit. The most commonly used surgical procedures are the percutaneous vertebroplasty with polymethyl methacrylate (PMMA) and the transpedicular screw fixationreconstruction. The aim of the present investigation is to summarize and elucidate the indications for the different types of spinal techniques, the potential complications and their treatment.
\end{abstract}

MATERIALS AND METHODS: 72 consecutive patients with compression spinal osteoporotic fractures treated in our clinic from January, 2009 to July, 2012 were analyzed. Of these, 38 patients (M14 / F24) at an average age of 66.8 years (54-80) underwent PV on 46 levels. All the fractures were without neurological deficit and classified as A1 type in Magerl. The remaining 34 patients (M5 / F29) at an average age of 67.3 years (56-85) underwent transpedicular screw spinal reconstruction addressing incomplete burst compression fractures in which the anterior two columns were damaged. A total of 40 levels were addressed. The indications for surgery were severe pain syndrome, neurological deficit, presence of bone fragments in the spinal canal or spinal instability.

RESULTS: In our series the VCF are most commonly located in the thoracolumbar (Th11-L2) segment of the spine - 75.6\%, (65/86) levels. In 7 patients 2 levels were treated in one settings, 2 patients had 3 levels treated and one patient 4 levels. Our PV subgroup showed very good outcome in $84.2 \%(32 / 38)$ of the patients. Due to migration of the cement in the spinal canal, two patients underwent surgical decompression. The results in the spinal instrumentation subgroup are good in 82.4\% (28/34) of the cases. In 6 of the instrumented cases a revision surgery was performed, due to progressing neurological deficit and persistent pain. The surgery addressed screw pull-outs and implant malposition/displacement.

CONCLUSION: Our results indicate that modern surgical treatment of osteoporotic patients with symptomatic VCF presents low incidence of complications and allows for a significant reduction of pain, spinal stability, improved quality of life and prolonged active life. PV is a minimally invasive procedure with a good effectiveness and uncommon complications that are clinically significant. The spinal instrumented reconstruction is indicated in cases of severe multilevel VCF, spinal instability, and compression of the neural structures.

Keywords: spinal instability, osteoporosis, complications, vertebroplasty, screw pull-out

Address for correspondence:

Svetoslav Kalevski

Medical University of Varna

55 Marin Drinov Str.,

9002 Varna, Bulgaria

e-mail:dr_kalevski@abv.bg

Received: May 6, 2016

Accepted: August 16, 2016

\section{BACKGROUND}

Osteoporosis is a systemic disease affecting mainly elderly patients. It is characterized with a reduction of bone density and impairment of the micro architectonics of the bone tissue and as a result of the above, low energy compression fractures tendency 
(1-5). In the last years, the frequently seen compression fractures in the elderly population became a serious problem for the health system. The number of vertebral compression fractures (VCF) significantly increases in the aging population. Hence the VCF become significant cause for pain, decreased quality of life, disability and even death. It is difficult to establish the real VCF rate due to the fact that only about one third will present with pain syndrome or impaired neurology. The remaining two-thirds will be asymptomatic (1,4-6). Furthermore, the diagnostic criteria are still not standardized and universally accepted. Most often, the fractures affect the thoracolumbar spine followed by middle and lower thoracic lumbar segments. Conventional treatment for VCF is non-operative and includes prolonged rest, NSAIDs and analgesics, wearing orthosis, rehabilitation, etc. (7-9). Although this treatment reduces the symptoms to some degree, patients are still exposed to a progressive functional limitation, insomnia, depression, gastrointestinal, and urological complications due to the prolonged drug intake and are prone to new compression fractures $(2,7)$.

Until recently, many spinal surgeons considered osteoporotic patients with scoliosis and osteoporotic vertebral fractures as not possible to operate on, even with neurological manifestations. With the advancement of technological innovations in the spinal reconstructive surgery - percutaneous vertebroplasty (PV) with PMMA kyphoplasty and open spinal instrumentation, more surgeons and patients are getting convinced of the benefits and the good results of the surgical treatment, especially when it comes to spinal instability or advancing neurological deficit (5). In modern spinal surgery, percutaneous vertebroplasty (PV) with PMMA is very well accepted, widely used, technically well developed and safe procedure with a high percentage of good results reaching up to $90 \%$ (9-13). The advantages of PV compared to the open spinal instrumentation are many, in terms of minimal invasiveness, fast pain relief, reconstruction of the height of the vertebrae, rapid hospital discharge, low cost, etc. (14). PV is indicated for patients with painful VCF without neurological symptoms, Magerl type A1, who have not responded well to the conservative treatment $(10,15,16)$. Absolute contraindication for PV is the presence of neurological symptoms, coagulopathy, severe heart and lung diseases, infections and febrility (12). The relative contraindication group includes significant loss of height of the vertebrae (75\%), a fracture involving the posterior wall cortex, displaced fragment, etc. (14).

Patients with neurological deficit due to compression of neural structures, as a result of migrated bone fragments after VCF, severe kyphoscoliosis, spinal instability, and most commonly - with intractable pain syndrome, usually necessitate open spinal instrumentation. Due to the decreased integrity of the bone trabeculae in the osteoporotic vertebral body, the implanted transpedicular screws are at risk of pull-out and resulting failure of the entire stabilizing implant $(3,17,18)$.

The advances of the operative techniques and spinal instrumentation, made better the purchase of the screws in the bone structures of the vertebrae possible. This includes improved geometry of the screws, use of additional laminar hooks and not least, augmentation of vertebral screws with bone cement (3,17-19).

In recent years, there have been many papers in the literature reporting on studies aimed at potential solutions of the problem with inadequate spinal fixation in osteoporotic patients. Despite some risk of extravasation and exothermic reaction, PMMA is the most widely used augmenting bone cement in spinal surgery today (18). The aim of our study is to summarize and discuss the indications for the different types of spinal techniques, potential complications, and methods for their treatment.

\section{MATERIALS AND METHODS:}

72 consecutive patients with compression spinal osteoporotic fractures treated in our clinic in the last three years were analyzed. Of these, 38 patients (M14 / F24) at an average age of 66.8 years (54-80) underwent PV on 46 levels. All the fractures were without neurological deficit and classified as A1 type (Magerl). An acute or chronic compression fracture with well expressed pain syndrome that had been difficult to control with the conservative measures was considered as an indication for PV. The aim of the treatment was to stabilize the segments, in order to stop the further collapse, to promote immediate mobilization of the patients and to achieve maximum reduction of the pain. PV was performed at one level in 32 patients, at two levels - in 4 patients and at three 
levels - in 2 patients. Prior to PV, all the patients were examined with standard X-rays, CT or MRI. The PV procedure was performed using a standard method with general anesthesia in a prone position. Under intraoperative X-ray control the index level is verified according to the preoperative imaging studies. The body of the collapsed vertebra is accessed through bilateral transpedicular access. Jamshidi needles with trocar are introduced into the front third of the vertebrae in a direction aiming at the midline. Initially,

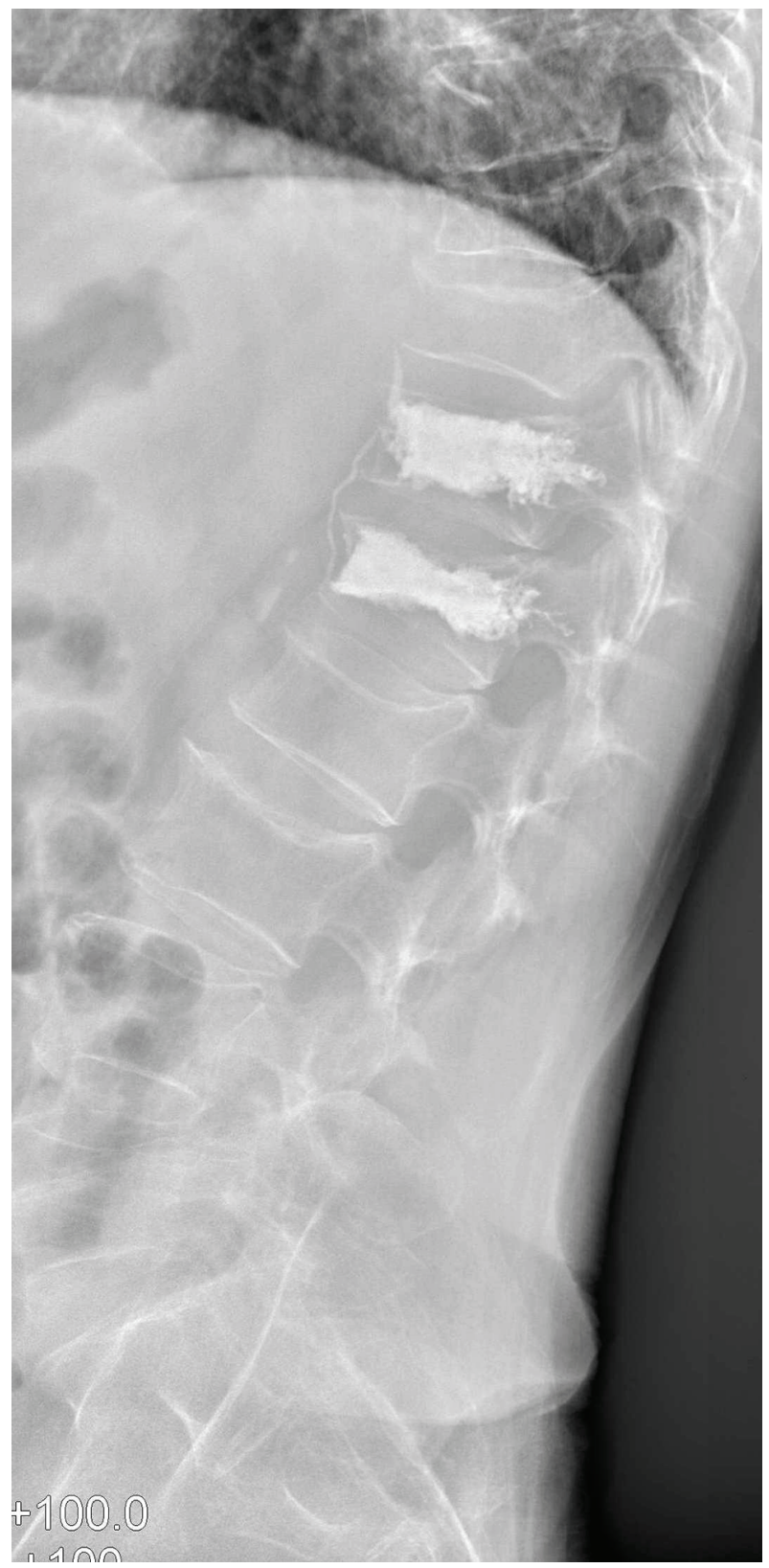

Fig. 1. PV with PMMA in osteoporotic VCF Magerl A1 at two adjacent levels - T12 and L1(F63)
$1 \mathrm{cc}$ of PMMA is injected in order to identify the direction of the potential leakage of the cement, paying more attention to the posterior wall of the vertebral body. If leakage is not detected at that point, the procedure is continued with an injection of between 2 and 10 more cc PMMA under moderate pressure (Fig. 1).

Extravasation of cement outside the body, particularly if it is in a larger amount, is an indication to stop the procedure. At the end of the procedure, before removing the Jamshidi cannula back, it is necessary to put the stilet back, in order to prevent leakage of cement in the soft tissues, which sometimes causes significant pain. In the early postoperative period, the neurovascular status and condition of the wound is monitored every 30 minutes. If no adverse events or complications are encountered, the patient is mobilized and if there is no need for any further treatment, the patient is discharged on the same day or no later than the following day. The dynamics of the pain syndrome is reflected before and after the procedure with a Visual Analog Scale (VAS). We have encountered cement extravasation into the spinal canal and adjacent neural foramen in two patients, causing new postoperative radicular syndrome corresponding to the level of the PA, without neurological deficit (Fig. 2).

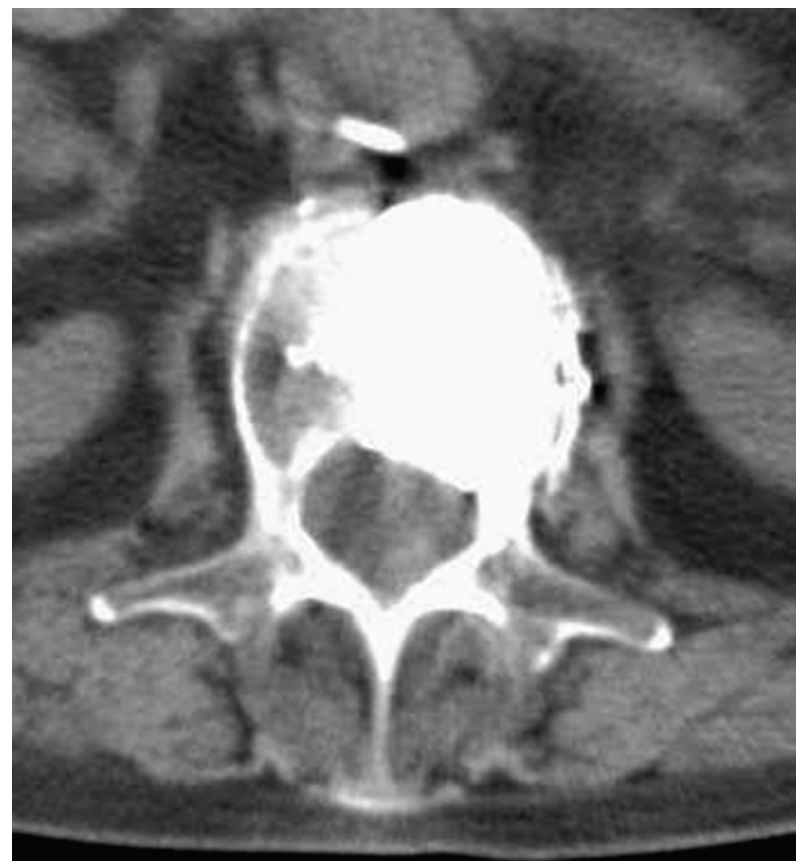

Fig. 2. PMMA leak towards the spinal canal and compression of the neural structures.(F/66) 
In either case, we made decompressive hemilaminectomy together with arthrotomy and foraminotomy and subsequent careful resection of the hardened PMMA located in the recess, as well as into the neuroforamen. The cement was aimed to be removed carefully after the pediculotomy, without excessive nerve root retraction. The remaining $34 \mathrm{pa}-$ tients (M5 / F29) at an average age of 67.3 years (5685) underwent transpedicular screw spinal reconstruction addressing incomplete burst compression fractures in which the anterior two columns were damaged. A total of 40 levels were addressed. Of these 34 patients, 30 had one level treated, three patients had 2 levels treated and one had four levels addressed. All patients were examined with standard X-rays, CT or MRI. The indications for surgery were severe pain syndrome, neurological deficit, a presence of bone fragments in the spinal canal or spinal instability. The pain syndrome was assessed pre- and postoperatively with VAS, and the neurological deficit was evaluated with the classification system of Frankel.

In the investigated cohort, 2 patients were with Frankel C, 20 were Frankel D, and 12 with Frankel E. The patients were divided into two groups: 1.Group I - patients with posterior instrumentation without augmentation with PMMA - 26; 2. Group II - patients with posterior instrumentation with augmentation with PMMA - 8.

The transpedicular fixation is performed by a standard procedure - the entry point of the screw is pointed just medially from the accessory process of the pedicle. In 25/30 patients, a short segment spinal fixation was performed to address the fracture - one level above and one level below the index level, while 9 patients underwent multisegment fixation addressing more than one fractured vertebra (Fig. 3). In 14 patients with CT and MRI data revealing compression of neural structures due to retropulsion of bone fragments or foraminal stenosis, the treatment comprised of decompressive laminectomy with foraminotomy.

The augmentation of the pedicular screws with PMMA is undertaken in the course of the surgical procedure, based on a decision of the operating surgeon, when the torque of the screws is considerably loose than usual. Before the insertion of the ce-

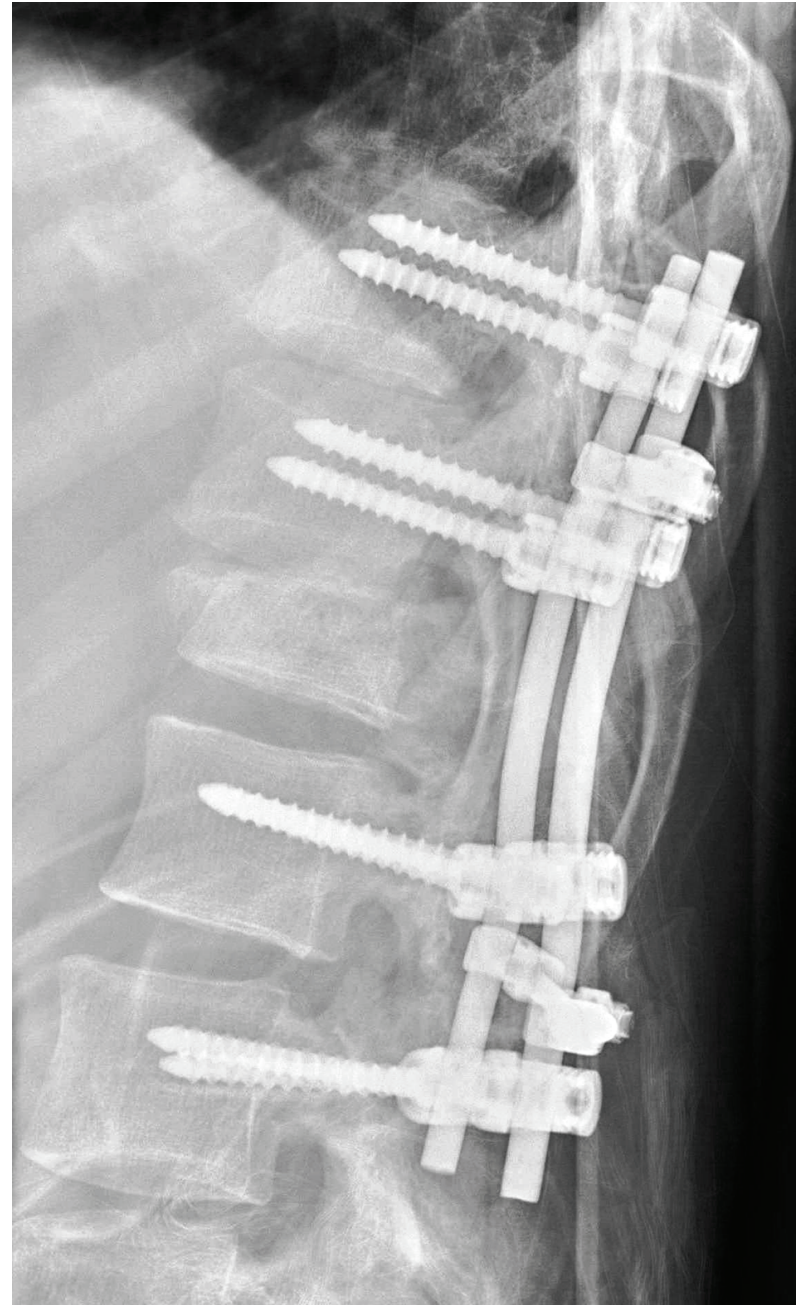

Fig. 3. Osteoporotic VCF L1. Laminectomy and foramenotomy at the index level. Multisegment transpedicular

fixation T11, T12 - L2, L3 (F66)

ment, the pedicle and the front wall of the vertebra is checked with a ball-tip-probe in order to rule out a damage and possible cement extravasation. Then a Jamshidi needle is inserted in the created canal in the pedicle and filled with PMMA. Before the insertion, the screw is also coated with about 1cc cement and screwed prior to polymerization (Fig. 4). The same procedure applies to the reimplantation of screws that had pulled out in a settings of instrumentation failure that we had in 6 of our cases (Fig. 5). In two of the cases with severe osteoporotic changes established during the course of open surgery, the vertebral bodies were previously augmented with PMMA and prior to the cement polymerization, the screws were implanted. 


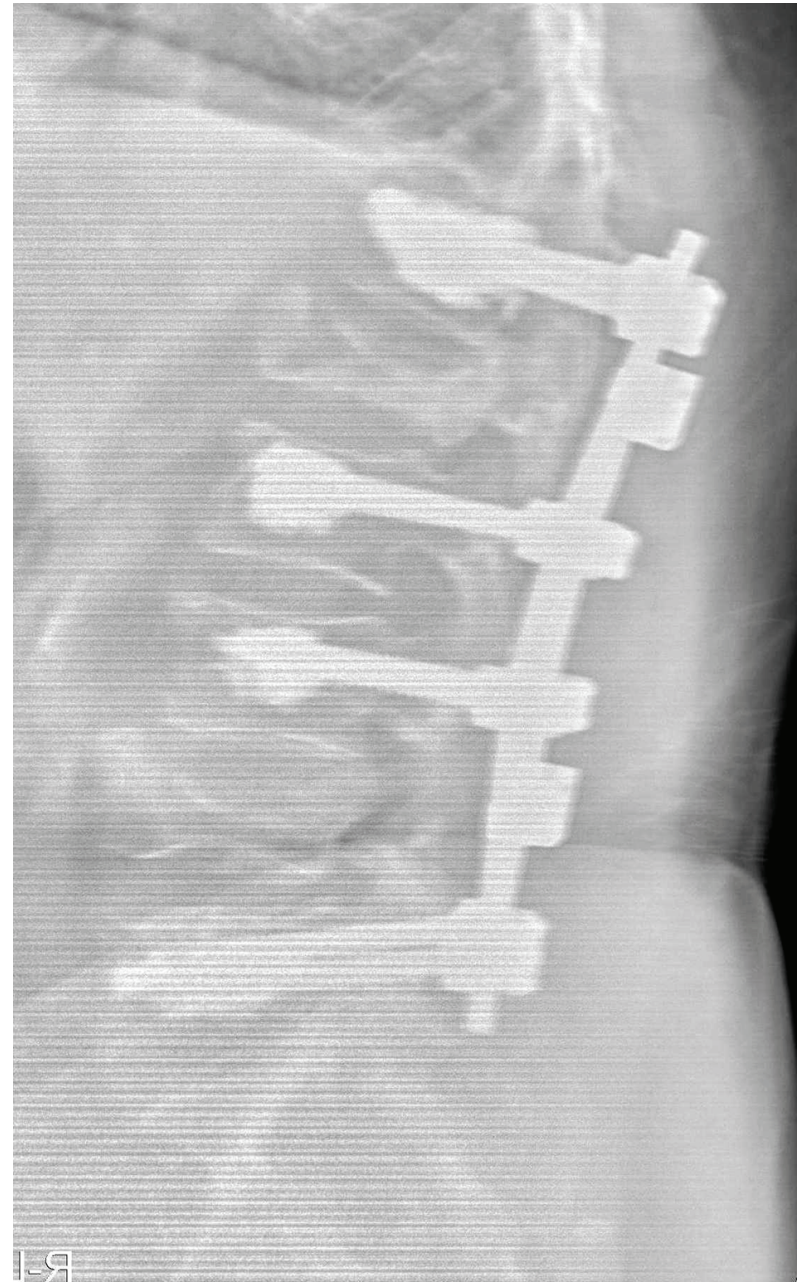

Fig. 4. Multilevel transpedicular fixation T12, L2, L3, L5 with PMMA augmentation (F72)

\section{RESULTS}

In our series, VCF are most commonly located in the thoracolumbar (Th11-L2) segment of the spine - 75.6\%, (65/86) levels, followed by mid-thoracic segment - 17.4\%, (15/86) levels, and finally, lower lumbar segment - 7\%, (6/86) levels. In 7 patients, 2 levels were treated in one settings, 2 patients had 3 levels treated and one patient - 4 levels. Our PV subgroup showed very good outcome in $84.2 \%(32 / 38)$ of patients. In the immediate postoperative period, a significant pain relief was registered in $30(78.9 \%)$ patients, moderate pain reduction in $6(15.8 \%)$. Only $2(5.3 \%)$ showed no improvement and even experienced deterioration. The mean preoperative and postoperative VAS values are respectively 7.5 and $3(\mathrm{p}<0.001)$. Symptomatic complications and adverse events were noted in 6 patients. Leakage of the cement out of the

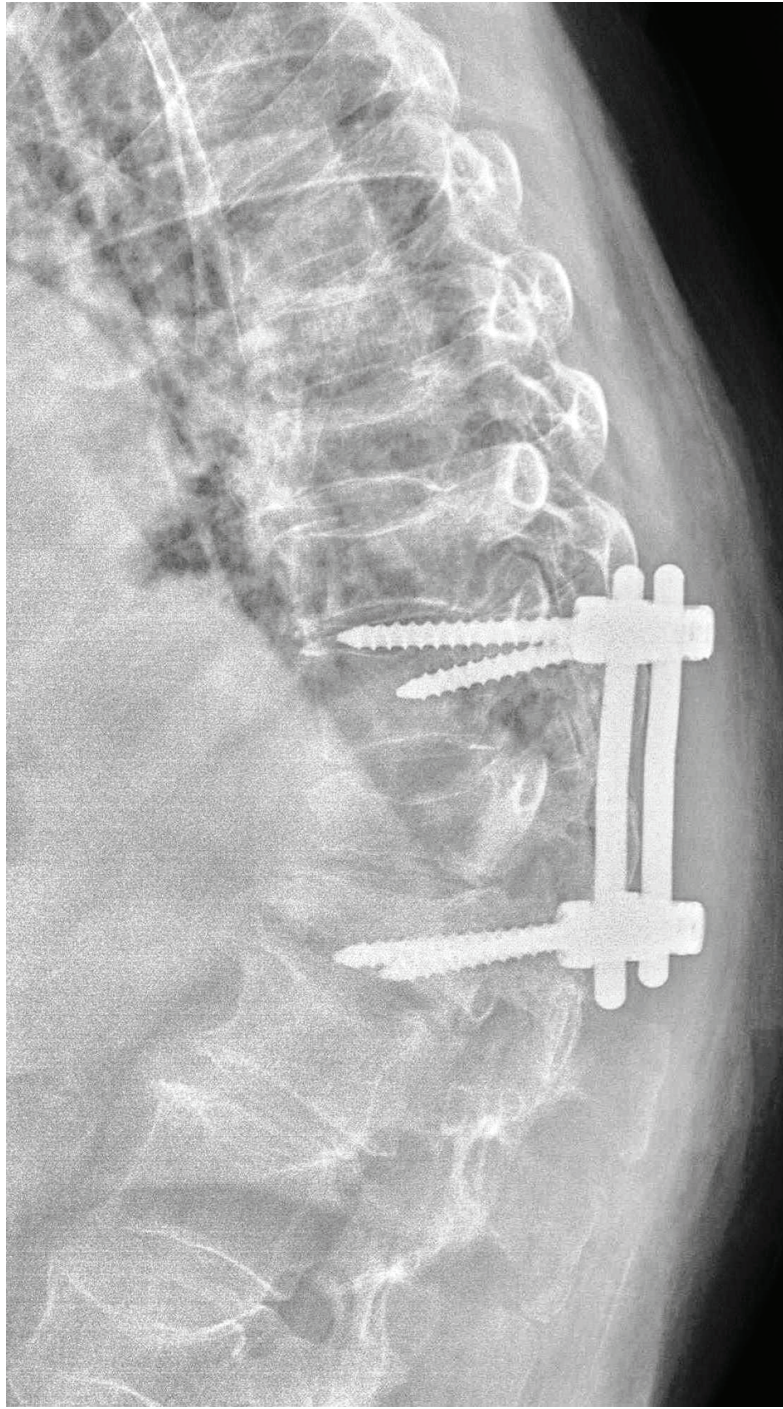

Fig. 5. Pedicular screws pull- out and malposition of the instrumentation due to impaired sagital balance and poor bone quality (F69)

vertebral body was registered at 20/46 levels (43.5\%). Of those, in 14 cases the extravasation was vascular (Fig. 5) and in 6 cases - nonvascular. Vascular leakage of the cement is seen mostly in the paravertebral venous system going out through the basivertebral artery. In one case, a pulmonary embolism with cement, without any clinical sequel, was established.

Nonvascular cement leakages are located along the needle track in 2 cases in the paravertebral soft tissue in 1 case, in the adjacent disk at the bottom endplate in 1 case, and in the spinal canal in 2 cases. In 4 patients with the above mentioned complications, the pain syndrome was controlled with con- 
servative treatment for 7-10 days. Due to a migration of the cement in the spinal canal, two patients underwent surgical decompression of the neural structures with a very good postoperative result. The results in the spinal instrumentation subgroup are good in $82.4 \%$ (28/34) of the cases, especially in those where the pain syndrome or the neurological deficit was due to spinal instability. Mean preoperative and postoperative VAS values were 8.5 and 3.5 , respectively $(\mathrm{p}<0.001)$.

In 6 of the instrumented cases, a revision surgery was performed, due to progressing neurological deficit and persistent pain. The surgery addressed screw pull-outs and implant malposition/displacement (Fig. 5). In the course of the augmentation procedures, no adverse events relating to cement extravasation were registered.

\section{DISCUSSION}

Osteoporotic VCF present a major clinical problem that leads to deformity and instability of the spine, persistent pain, reduced pulmonary function and mobility, hence an overall increase in disability and mortality in elderly patients $(9,20)$. The pathophysiology of osteoporosis is not yet completely revealed. It is being classified as primary and secondary. The primary is subdivided into two subtypes: Type I - in women 3-8 years after menopause, being a result of estrogen deficiency and the second subtype - senile (Type II), mostly seen among women and men over 70 years of age due to calcium intake deficiency (6). Secondary osteoporosis includes cases of unknown origin and also those who have been immobilized for long periods or time or being treated with corticosteroids long term (1).

Quite often, the undertaken conservative or surgical treatment proves to be inadequate for the case. Still, there is no consensus regarding both the indications for the surgical treatment itself and the type of the spinal reconstruction, as well as the time frames in which it should be considered (5). Analysis of the current literature shows different opinions coming from different authors regarding the best timing for PV. Some believe that one must wait a period of three months after a failed conservative treatment and then move to PV (14). Other authors $(5,10)$, and our opinion as well, think that the waiting period should be shorter - one month after the fracture.
Although there is a higher risk of cement extravasation, a shorter period avoids bone ischemia and possible progression of the vertebral collapse. Although many authors describe complications and adverse effects after PV $(5,9,11,14-16,20,21)$, the reconstruction of the anterior column gains good results in up to $90 \%$ of the cases, while the clinically significant complications are 1-3\% (22). In this respect, we prefer PV in patients with VCF where selection was made following strict indications. We believe that this is the reason why in our series there were no severe complications, such as procedure-related infections, permanent neurological deficits or death, although we have a case of pulmonary embolism with cement, also a case with massive extravasation in the vertebrobasilar vein, in the paravertebral venous system, which fortunately was without clinical sequelae.

The mild cases of radiculopathy or axial vertebral pain associated with unwanted migration of cement are usually transient and relatively easily controlled with analgesics for several days. There is a wide variety of surgical techniques proposed for the treatment of osteoporotic patients with neurological deficits and spinal instability $(3,4,17,18,22,23)$. Some authors are proponents of the anterior decompression and reconstruction, while others $(22,23)$ believe that stand-alone anterior decompression and fusion are not enough and one will need additional posterior instrumentation. Furthermore, the anterior access requires longer operating time and carries a high risk of lung and abdominal complications, especially when it comes to elderly patients. Another drawback of the anterior access is that it does not provide an access to the posterior structures that are usually degenerative and hypertrophied in this age group and that of course further contributes for the narrowing of the spinal canal and the potential deterioration of the neurological impairment (22).

The mild cases of radiculopathy or axial vertebral pain associated with unwanted migration of cement are usually transient and relatively easily controlled with analgesics for several days. There is a wide variety of surgical techniques proposed for the treatment of osteoporotic patients with neurological deficits and spinal instability $(3,4,17,18,22,23)$. Some authors are proponents of the anterior decompression and reconstruction, while others $(22,23)$ believe that stand-alone anterior decompression and fusion 
are not enough and one will need additional posterior instrumentation. Furthermore, the anterior access requires longer operating time and carries a high risk of lung and abdominal complications, especially when it comes to elderly patients. Another drawback of the anterior access is that it does not provide an access to the posterior structures that are usually degenerative and hypertrophied in this age group and that of course further contributes for the narrowing of the spinal canal and the potential deterioration of the neurological impairment (22).

The posterior and posterolateral surgical techniques and instrumentation provide much better decompression and spinal reconstruction $(24,25)$. Furthermore, the back surgery allows decompression of more levels at the same time and in the same position. And last but not least, spinal surgeons are more familiar with the posterior approaches (22). In choosing the surgical procedure, the most important step is the evaluation of the condition of each patient, taking into account a number of important factors: (1) the general condition of the patient, (2) the type, level and number of fractures, (3) the degree of osteoporosis, and (4) the best possible technique with which to perform the decompression and to correct kyphosis (4). Direct decompression of neural structures and spinal reconstruction are the best solution in the treatment of patients with unstable VCF with retropulsion of fragments in the vertebral canal, associated with neurological deficit $(4,22,23)$. It is known that the main problem of the posterior reconstructive surgery in osteoporotic patients is the lack of a healthy bone structure especially in the anterior column $(3,17,18)$. Therefore, it is very difficult to provide a stable connection between the bone and the implant and instrumentation often fails, particularly in elderly kyphotic patients who are corrected without strengthening the anterior column. Most often, with a minimal effort while bending, the pedicular screws from the upper segments pull out, and this leads to spinal instability, and strong and constant pain. Over the last decade, after the introduction of PA with PMMA, the augmentation of the anterior column became possible with good clinical results $(2,5,6,9,11-$ 14). At a later stage, there were many variations proposed for the augmentation of the vertebral body and the pedicular screws in order to increase the pull out resistance and the total consolidation of the instru- mentation to an extent sometimes greater than that of the normal spine $(1,3,4,18,22,23,25)$. In our opinion, the augmentation of the vertebral body before the implantation of screws is a good physiological solution in osteoporotic patients. Our results indicate that the cases of monosegment reconstruction without augmentation with PMMA were successful when the neurological deficit or pain syndrome were mainly the result of spinal instability, rather than a result of bone compression or deformation. The multilevel fractures, especially those with pseudarthrosis are subjected to a greater risk of implant failure and need multisegment spinal reconstructions with augmentation. Restoration of failed posterior instrumentation also requires augmentation of the vertebrae and screws before reimplantation.

\section{CONCLUSION}

Our results indicate that modern surgical treatment of osteoporotic patients with symptomatic VCF presents low incidence of complications and allows for a significant reduction of pain, spinal stability, improved quality of life and prolonged active life.

\section{REFERENCES}

1. Wu C.-T. et al. Classification of symptomatic osteoporotic compression fractures of the thoracic and lumbar spine. Journal of Clinical Neuroscience 2006;13:31-38.

2. Afzal S, Dhar S et al. Percutaneous Vertebroplasty for Osteoporotic Fractures. Pain Physician 2007; 10:559-563.

3. Sawakami K, Yamazaki A et al. Polymethylmethacrylate Augmentation of Pedicle Screws Increases the Initial Fixation in Osteoporotic Spine Patients. J Spinal Disord Tech 2011;(00)00:1-7.

4. Long HQ, Wan Y, Zhang X, Liu SY, Li FB. Two kinds of posterior approach for Kьmmell's disease after osteoporotic thoracolumbar fracture. Chinese Journal of Traumatology 2009;12(3):142-147.

5. Lovi A, Teli M, Ortolina A et al. Vertebroplasty and kyphoplasty: complementary techniques for the treatment of painful osteoporotic vertebral compression fractures. A prospective non-randomised study on 154 patients. Eur Spine J 2009;18 (Suppl 1):S95-S101.

6. Kostuik JP. Fractures of the cervical, thoracic, and lumbar spine. Marcel Dekker New York; 2002:635-654. 
7. Chen L-H. Current Status of Vertebroplasty for Osteoporotic Compression Fracture. Chang Gung Med J 2011;34:352-9.

8. Gardner M J, Demetrakopoulos D, Shindle MK et al. Osteoporosis and Skeletal Fractures. HSSJ 2006; 2: 62-69.

9. Lieberman I. Vertebral Augmentation for Osteoporotic and Osteolytic Vertebral Compression Fractures: Vertebroplasty and Kyphoplasty Advances in Spinal Stabilization. Basel 2003;16: 240-250

10. Chen L-H et al .Minimally Invasive Treatment of Osteoporotic Vertebral Compression Fracture. Chang Gung Med J 2004;27:261-7.

11. Hiwatashi A, Westesson P-LA. Vertebroplasty for Osteoporotic Fractures with Spinal Canal Compromise. Am J Neuroradiol. 2007;28:690 -92.

12. Mohit AA, Douglas O. Percutaneous Vertebral Augmentation in Pathologic Fractures. Curr Opin Orthop 2007;18:221-225.

13. Brodano GB, Amendola L, Martikos K et al. Vertebroplasty: benefits are more than risks in selected and evidence-based informed patients. A retrospective study of 59 cases. Eur Spine J 2011; 20:1265-1271.

14. Layton KF, Thielen KR, Koch CA et al. Vertebroplasty, First 1000 Levels of a Single Center: Evaluation of the Outcomes and Complications. Am J Neuroradiol. 2007;28:683- 89.

15. Tanigawa N, Kariya S Komemushi A et al. Percutaneous Vertebroplasty for Osteoporotic Compression Fractures: Long-Term Evaluation of the Technical and Clinical Outcomes. AJR 2011;196:1415-1418.

16. Yang S-C, Chen W-J et al. Revision strategies for complications and failure of vertebroplasties. Eur Spine J 2008;17:982-988.

17. Aydogan M, Ozturk C et al. The Pedicle Screw Fixation With Vertebroplasty Augmentation in the Surgical Treatment of the Severe Osteoporotic Spines. J Spinal Disord Tech . 2009; (22) 6:444-7.

18. Burval DJ, McLain RF et al. Primary Pedicle Screw Augmentation in Osteoporotic Lumbar Vertebrae. SPINE 2007;(32) 10:1077-1083

19. Sudo $\mathrm{H}$, Ito $\mathrm{M}$, Abumi K et al. One-stage posterior instrumentation surgery for the treatment of osteoporotic vertebral collapse with neurological deficits. Eur Spine J 2010;19:907-915.
20. Li K-C, Li A. F.-Y. et al. Transpedicle body augmenter in painful osteoporotic compression fractures. Eur Spine J 2007;16:589-598.

21. Yeom J. S., Kim W. J., Choy W. S. et al. Leakage of cement in percutaneous transpedicular vertebroplasty for painful osteoporotic compression fractures. J Bone Joint Surg [Br] 2003;85-B:83-9.

22. Takahashi J, Kamimura $M$ et al. Anterior Vertebral Reconstruction Surgery using Polymethylmethacrylate and Posterior Spinal Short Fusion through the Posterior Approach for Osteoporotic Vertebral Pseudoarthrosis. The Open Spine Journal 2010;2:29-34.

23. Ataka H, Tanno T, Yamazaki M. Posterior instrumented fusion without neural decompression for incomplete neurological deficits following vertebral collapse in the osteoporotic thoracolumbar spine. Eur Spine J. 2009;18:69-76.

24. Hu M.-H. Wu H. T. H. Chang M.-C. Polymethylmethacrylate augmentation of the pedicle screw: the cement distribution in the vertebral body. Eur Spine J 2011; 20:1281-1288.

25. Pinera A.R, Duran C. et al..Instrumented lumbar arthrodesis in elderly patients: prospective study using cannulated cemented pedicle screw instrumentation. Eur Spine J 2011; 20 (3):S408-S414 